\title{
OSELTAMIVIR-RESISTANT INFLUENZA A(H1N1) VIRUSES DETECTED IN EUROPE DURING SEASON 2007-8 HAD EPIDEMIOLOGIC AND CLINICAL CHARACTERISTICS SIMILAR TO CO-CIRCULATING SUSCEPTIBLE A(H1N1) VIRUSES
}

\author{
B C Ciancio (bruno.ciancio@ecdc.europa.eu) ${ }^{1}$, T J Meerhoff², P Kramarz', I Bonmarin ${ }^{3}$, K Borgen $^{4}$, C A Boucher ${ }^{5}$, U Buchholz ${ }^{6}$, \\ S Buda 6 , F Dijkstra 7 , S Dudman ${ }^{4}$, S Duwe ${ }^{5}$, S H Hauge ${ }^{4}$, 0 Hungnes $^{4}$, A Meijer 7 , J Mossong ${ }^{8}$, W J Paget ${ }^{2}$, N Phin ${ }^{9}$, M van der \\ Sande7, B Schweiger ${ }^{6}$, A Nicoll ${ }^{1}$ \\ 1. European Centre for Disease Prevention and Control (ECDC), Stockholm, Sweden \\ 2. The Netherlands Institute for Health Services Research (NIVEL), Utrecht, the Netherlands \\ 3. Institut de veille sanitaire (InVS), Paris, France \\ 4. The Norwegian Institute of Public Health (Folkehelseinstituttet), Oslo, Norway \\ 5. Erasmus Medical Centre, Rotterdam, the Netherlands \\ 6. Robert-Koch-Institut (RKI), Berlin, Germany \\ 7. National Institute for Public Health and the Environment (RIVM), Bilthoven, the Netherlands \\ 8. Laboratoire National de Santé, Luxembourg \\ 9. Health Protection Agency, London, United Kingdom
}

This article was published on 19 November 2009.

Citation style for this article: Ciancio BC, Meerhoff TJ, Kramarz P, Bonmarin I, Borgen K, Boucher CA, Buchholz U, Buda S, Dijkstra F, Dudman S, Duwe S, Hauge SH, Hungnes 0, Meijer A, Mossong J, Paget WJ, Phin N, van der Sande M, Schweiger B, Nicoll A. Oseltamivir-resistant influenza A(H1N1) viruses detected in Europe during season 2007-8 had epidemiologic and clinical characteristics similar to co-circulating susceptible A(H1N1) viruses . Euro Surveill. 2009;14(46):pii=19412. Available online: http://www. eurosurveillance.org/ViewArticle.aspx?ArticleId=19412

During the 2007-08 influenza season, high levels of oseltamivir resistance were detected among influenza $A(H 1 N 1)$ viruses in a number of European countries. We used surveillance data to describe influenza $A(\mathrm{H} 1 \mathrm{~N} 1)$ cases for whom antiviral resistance testing was performed. We pooled data from national studies to identify possible risk factors for infection with a resistant virus and to ascertain whether such infections led to influenza illness of different severity. Information on demographic and clinical variables was obtained from patients or their physicians. Odds ratios for infection with an oseltamivir resistant virus and relative risks for developing certain clinical outcomes were computed and adjusted through multivariable analysis. Overall, 727 (24.3\%) of 2,992 tested influenza $\mathrm{A}(\mathrm{H} 1 \mathrm{~N} 1)$ viruses from 22 of 30 European countries were oseltamivir-resistant. Levels of resistance ranged from $1 \%$ in Italy to $67 \%$ in Norway. Five countries provided detailed case-based data on 373 oseltamivir resistant and 796 susceptible cases. By multivariable analysis, none of the analysed factors was significantly associated with an increased risk of infection with an oseltamivir-resistant virus. Similarly, infection with an oseltamivirresistant virus was not significantly associated with a different risk of pneumonia, hospitalisation or any clinical complication. The large-scale emergence of oseltamivir-resistant viruses in Europe calls for a review of guidelines for influenza treatment.

\section{Introduction}

In Europe, virological surveillance of antiviral susceptibility of influenza viruses has been performed since 2004 through the European Union (EU)-funded European Surveillance Network for Vigilance against Viral Resistance (VIRGIL), in collaboration with the European Influenza Surveillance Scheme (EISS), the World Health Organization (WHO) and national influenza centres (NICs) [1]. In January 2008 this surveillance system started to detect significant proportions of oseltamivir-resistant viruses among influenza $A(\mathrm{H} 1 \mathrm{~N} 1)$ specimens collected in several European countries from November 2007 onwards [2]. This was associated with a histidine to tyrosine mutation at residue 275 of the neuraminidase protein ( $\mathrm{H} 275 \mathrm{Y}$ or $\mathrm{H} 274 \mathrm{Y}$ in $\mathrm{N} 2$ numbering), which is known to confer high level resistance to the neuraminidase inhibitor oseltamivir [3]. Oseltamivir resistance was confirmed in most EU countries as more influenza $\mathrm{A}(\mathrm{H} 1 \mathrm{~N} 1)$ viruses were isolated and tested, although at very different levels ranging from under $2 \%$ of all influenza $A(\mathrm{H} 1 \mathrm{~N} 1)$ viruses tested in Italy and Spain to over $40 \%$ in Belgium, Estonia, France and Norway by the end of the 2007-8 influenza season $[4,5]$. These differences, however, were also influenced by the time during the season when specimens were collected and the number of influenza $A(H 1 N 1)$ viruses tested for oseltamivir susceptibility in each country [6]. The wide circulation as well as outbreaks of oseltamivir-resistant viruses, together with a rise in resistance proportions throughout the season indicated that influenza $\mathrm{A}(\mathrm{H} 1 \mathrm{~N} 1) \mathrm{H} 275 \mathrm{Y}$-mutated strains were fit and transmissible [6]. This was supported by the absence of correlation between oseltamivir resistance and exposure to oseltamivir at population level [7]. However, it was unclear whether there were any factors favouring infection with an oseltamivir-resistant virus and whether such an infection would affect the clinical course of influenza illness with or without treatment.

In order to obtain additional data on the characteristics of patients infected with influenza $A(H 1 N 1)$ viruses, the EISS and VIRGIL coordination centres rapidly set up an enhanced 
surveillance system requesting the European NICs to report for confirmed influenza $\mathrm{A}(\mathrm{H} 1 \mathrm{~N} 1)$-infected patients additional information (such as clinical outcome and exposure to antivirals) to that already routinely collected. Furthermore, a number of countries in the EU and European Economic Area (EEA) conducted specific epidemiological investigations based on a general protocol developed by the European Centre for Disease Prevention and Control (ECDC) in collaboration with some EU countries with the following objectives:

- To identify risk factors for infection with an oseltamivir-resistant versus an oseltamivir-susceptible influenza $A(\mathrm{H} 1 \mathrm{~N} 1)$ virus during the 2007-8 influenza season.

- To assess whether patients infected by an oseltamivir-resistant influenza $A(H 1 N 1)$ virus had a different risk of a severe clinical outcome than patients infected by an oseltamivir-susceptible influenza $A(\mathrm{H} 1 \mathrm{~N} 1)$ virus.

The study hypothesis was that oseltamivir-resistant influenza $A(H 1 N 1)$ viruses emerged during the $2007-8$ season were different from co-circulating oseltamivir-susceptible influenza $A(H 1 N 1)$ viruses in terms of risk factors for infection and severity of illness.

This article reports on the descriptive analysis of data from the enhanced surveillance and on the analysis of the pooled data from the national epidemiological studies.

\section{Methods}

\section{Surveillance data}

The descriptive analysis of influenza surveillance data concerns information collected during the season 2007-8 from week 40/2007 to week 20/2008 in countries participating in EISS. National surveillance systems collect standard case-based epidemiological information for all patients undergoing clinical sampling for laboratory confirmation. However, this information is not routinely reported to EISS. Laboratory confirmation is carried out for surveillance purposes on a subset of individuals presenting with influenza-like illness (ILI) and/or symptoms of acute respiratory infection (ARI) to one of the sentinel physicians participating in the national influenza surveillance. The selection of patients with ILI or ARI undergoing virological testing can be either random/systematic, as recommended by EISS, or left to the physician's clinical judgement [8]. Virological testing is usually performed at the NICs, which are WHO-recognised laboratories for influenza and in Europe collaborate within the Community Network of Reference Laboratories (CNRL) for human influenza [9]. The sentinel physicians are part of national networks that intend to cover a representative sample of the general population. Moreover, case-based information is collected nationally on patients tested for influenza as part of the individual clinical management (nonsentinel samples). Such samples cover a heterogeneous group of individuals including hospitalised patients who are likely to have experienced a more severe influenza illness. In Norway, however, both non-sentinel and sentinel specimens are collected mainly from patients presenting to the primary healthcare system. Additional information on the organisation and functioning of virological influenza surveillance in Europe can be found elsewhere [10].

During the season 2007-8, when higher than expected levels of oseltamivir resistance were detected in influenza $A(\mathrm{H} 1 \mathrm{~N} 1)$ viruses in many European countries, the data routinely collected by EISS and VIRGIL was expanded to include the following additional information: oseltamivir susceptibility, age, gender, geographic location, hospital or community-based, date of specimen collection, date of disease onset, exposure to antivirals of the patient or household contact (in the 14 days preceding onset of illness), influenza vaccination status, and whether complications, hospitalisations or death occurred in the 14 days following onset of illness. Oseltamivir susceptibility was determined phenotypically or by sequencing or by both, as described elsewhere [6]. Data were uploaded during the season and were downloaded on 19 August 2008. The descriptive virological surveillance data presented in this paper might differ slightly from those presented previously [6], as data for the present paper were downloaded one month later and countries could have updated the database since then. In addition, the weeks included in reference [6] (weeks 40-19) differed by one week from the data presented in this paper (weeks 40-20). A descriptive analysis was carried out and individual characteristics were assessed.

Some European countries experiencing high levels of oseltamivir resistance collected additional information on influenza $A(H 1 N 1)$ cases by retrospectively interviewing patients and/or their physicians. The ECDC supported and coordinated such studies by providing a study protocol and organising three meetings as well as regular teleconferences with the study group. To increase the efficiency and timeliness of a European study, only those countries were invited to participate in which at least 50 virus isolates had been tested for antiviral resistance and some level of oseltamivir resistance had been detected as of February 2008. Of the six countries that met this criterion for inclusion, five (Germany, Luxembourg, the Netherlands, Norway and the United Kingdom (UK)) agreed to participate and to provide their databases for a pooled analysis by ECDC.

\section{Epidemiological studies}

Questionnaires and study procedures developed by each of the five participating countries were submitted to the ECDC in order to identify common variables for the joint analysis. In all participating countries, the study population included all individuals diagnosed with an influenza $A(H 1 N 1)$ virus infection between week 40/2007 and week 20/2008 for whom antiviral susceptibility testing was performed and for whom it was clear whether the specimens came from sentinel or non-sentinel sources.

Analysis of risk factors for infection with resistant virus

To identify risk factors for infection with an oseltamivir-resistant influenza $A(\mathrm{H} 1 \mathrm{~N} 1)$ virus, a nested case control approach was chosen within the cohort of subjects with laboratory-confirmed influenza $A(H 1 N 1)$ infection. Cases were defined as individuals with laboratory-confirmed influenza $\mathrm{A}(\mathrm{H} 1 \mathrm{~N} 1)$ infection whose isolates showed phenotypic (IC50 level) or genetic (H275Y mutation) markers of oseltamivir resistance, and controls were defined as individuals with laboratory-confirmed influenza $A(\mathrm{H} 1 \mathrm{~N} 1)$ infection whose isolates were susceptible to oseltamivir by either phenotypic or genetic analysis. Information was collected for cases and controls on age, sex, country of residence, location of initial sampling (sentinel versus non-sentinel), pre-existing medical conditions, influenza vaccination status, antiviral exposure (i.e. prophylaxis or treatment in the 14 days preceding symptom onset) and travel history within 10 days before symptom onset.

\section{Analysis of outcomes of infection with resistant virus}

To assess whether patients infected by oseltamivir-resistant influenza $A(H 1 N 1)$ virus were at higher risk of a severe clinical outcome than patients infected by oseltamivir-susceptible influenza 
$\mathrm{A}(\mathrm{H} 1 \mathrm{~N} 1)$ virus, a cohort approach was chosen, with cases and controls as the exposed and the unexposed subjects, respectively. The outcomes investigated were symptoms at presentation, hospitalisation for any cause related to influenza, pneumonia, death, and any other clinical complication attributable to influenza virus infection.

\section{Data collection}

Retrospective data for the case control analysis and follow-up information for the cohort analysis were collected using slightly different methods and data sources in the different countries. In Germany a subset and in Luxembourg all patients with a confirmed influenza $A(H 1 N 1)$ infection were contacted by local or national public health offices and administered a questionnaire by telephone (Germany) or mail (Luxembourg) in addition to the information already retrieved from the routine surveillance datasets. In the Netherlands, all sentinel physicians and virologists (and subsequently the treating clinicians in the hospitals) who had provided specimens positive for influenza $A(\mathrm{H} 1 \mathrm{~N} 1)$ were contacted by the national public health institute and sent a questionnaire by mail. Those not responding were contacted by telephone. In Norway, general practitioners (GPs) and clinicians in hospitals who had reported an influenza $A(\mathrm{H} 1 \mathrm{~N} 1)$ case to the NIC were contacted by the national public health institute and administered

TA B L E 1

Influenza detections and oseltamivir resistance of influenza $\mathrm{A}(\mathrm{H} 1 \mathrm{~N} 1)$ viruses in countries reporting data to EISS and VIRGIL during the 2007-8 influenza season (surveillance database)

\begin{tabular}{|c|c|c|c|c|c|c|c|}
\hline Country & $\begin{array}{l}\text { Specimens tested } \\
\text { positive for } \\
\text { influenza virus }\end{array}$ & $\begin{array}{l}\text { Influenza A } \\
\text { detections; (\% in } \\
\text { brackets) }\end{array}$ & $\begin{array}{l}\text { Influenza } A(H 1) \\
\text { virus detections } \\
\text { / subtyped } \\
\text { viruses }\end{array}$ & $\begin{array}{l}\text { Influenza A(H1N1) } \\
\text { viruses tested } \\
\text { for oseltamivir } \\
\text { resistance }\end{array}$ & $\begin{array}{c}\text { InflluenzaA(H1N1) } \\
\text { viruses resistant } \\
\text { to oseltamivir } \\
\text { (\% in brackets) }\end{array}$ & $\begin{array}{l}\text { Proportion of } \\
\text { resistant viruses } \\
\text { detected by } \\
\text { sentinel sources }\end{array}$ & $\begin{array}{l}\text { Case- based } \\
\text { clinical data } \\
\text { available in } \\
\text { surveitlance } \\
\text { database (yes/no) }\end{array}$ \\
\hline Austria & 531 & $457(86)$ & $262 / 262$ & 164 & $12(7.3)$ & 100 & Yes \\
\hline Belgium & 918 & $596(65)$ & $312 / 318$ & 32 & $17(53.1)$ & 100 & Yes \\
\hline Bulgaria & 21 & $16(76)$ & $16 / 16$ & 9 & 0 & n.a. & n.a. \\
\hline Croatia & 176 & $113(64)$ & 91/91 & 6 & 0 & n.a. & n.a. \\
\hline Czech Republic & 262 & $176(67)$ & $135 / 135$ & 24 & 0 & n.a. & n.a. \\
\hline Denmark & 306 & $203(66)$ & $182 / 196$ & 45 & $2(4.4)$ & n.a. & Yes \\
\hline Estonia & 244 & $207(58)$ & $137 / 198$ & 7 & $3(42.9)$ & 100 & Yes \\
\hline Finland & 209 & $165(79)$ & $69 / 138$ & 13 & $3(23.1)$ & n.a. & No \\
\hline France & 2,887 & $1,820(63)$ & $255 / 267$ & 496 & $231(46.6)$ & n.a. & No \\
\hline Germany & 2,199 & $1,098(50)$ & $1,002 / 1,042$ & 505 & 66 (13.1) & 79 & Yes \\
\hline Greece & 213 & $140(66)$ & $136 / 136$ & 65 & $7(10.8)$ & 80 & Yes \\
\hline Hungary & 212 & $173(82)$ & $154 / 154$ & 11 & 0 & n.a. & n.a. \\
\hline Ireland & 211 & $110(52)$ & $74 / 81$ & 63 & $7(11.1)$ & 100 & Yes \\
\hline Italy & 210 & $111(53)$ & $49 / 62$ & 106 & $1(0.9)$ & 0 & Yes \\
\hline Latvia & 608 & 586 (96) & $340 / 343$ & 15 & 0 & n.a. & n.a. \\
\hline Luxembourg & 463 & $264(57)$ & $18 / 18$ & 227 & $59(26.0)$ & 78 & Yes \\
\hline Netherlands & 443 & $232(52)$ & $165 / 191$ & 171 & $46(26.9)$ & 30 & Yes \\
\hline Norway & 856 & 466 (54) & $296 / 313$ & 273 & $184(67.4)$ & 20 & Yes \\
\hline Poland & 88 & $53(60)$ & $24 / 24$ & 10 & $1(10.0)$ & n.a. & No \\
\hline Portugal & 118 & $52(44)$ & $52 / 52$ & 29 & $6(20.7)$ & n.a. & No \\
\hline Romania & 482 & $372(77)$ & $361 / 372$ & 49 & $4(8.2)$ & 100 & Yes \\
\hline Serbia & 63 & $60(95)$ & $60 / 60$ & 18 & 0 & n.a. & n.a. \\
\hline Slovakia & 198 & $159(80)$ & $119 / 120$ & 14 & 0 & n.a. & n.a. \\
\hline Slovenia & 269 & $252(94)$ & $173 / 174$ & 28 & $1(3.6)$ & n.a. & No \\
\hline Spain & 1,738 & 805 (46) & $539 / 564$ & 106 & $2(1.9)$ & 100 & Yes \\
\hline Sweden & 1,318 & $487(37)$ & $71 / 82$ & 36 & $4(11.1)$ & 0 & Yes \\
\hline Switzerland & 620 & 394 (64) & $128 / 135$ & 53 & $10(18.9)$ & 90 & Yes \\
\hline Turkey & n.a. & n.a. & n.a. & 3 & 0 & n.a. & n.a. \\
\hline Ukraine & 128 & $85(66)$ & $35 / 35$ & 67 & $23(34.3)$ & n.a. & No \\
\hline United Kingdom & 1,887 & $1,044(55)$ & $475 / 545$ & 347 & $38(11.0)$ & 29 & Yes \\
\hline Total & 17,878 & 10,471 (59) & $5,765 / 6,003$ & 2,992 & $727(24.3)$ & & \\
\hline
\end{tabular}

Countries marked in bold were included in the analytical study.

EEA: European economic area; EFTA: European Free Trade Association; EU: European Union; n.a.: not available.

a Data available in EISS database on 8 July 2008.

b Data extracted 27 August 2008 from the EISS-VIRGIL. A number of countries tested all influenza A(H1N1) and influenza A viruses for oseltamivir

resistance by pyro-sequencing. Some samples were not definitely proven to be H1 subtype, therefore the number of H1 virus detections can be lower than

the number of tests for resistance. 
T A B L E 2

Risk factors for being infected with an oseltamivir-resistant virus, data from five EU and EEA/EFTA countries, 2007-8 influenza season $(n=1,169)$

\begin{tabular}{|c|c|c|c|c|}
\hline Factor & Categories & $\begin{array}{l}\% \text { oseltamivir-resistant virus }{ }^{\mathrm{a}, \mathrm{b}} \\
\mathrm{N}: 373(1,169)\end{array}$ & Crude odds ratio (95\% CI) & Adjusted odds ratio $(95 \% \mathrm{CI})^{\mathrm{c}, \mathrm{d}}$ \\
\hline \multirow{3}{*}{ Age in years } & $0-17$ & $28(572)$ & 1 & 1 \\
\hline & $18-64$ & $43(439)$ & $1.93(1.49-2.51)$ & $1.39(1.01-1.91)$ \\
\hline & $>65$ & $60(10)$ & $3.76(1.05-13.51)$ & $2.33(0.52-10.47)$ \\
\hline \multirow{2}{*}{ Sex } & Female & $36(536)$ & 1 & \multirow{2}{*}{ n.i. } \\
\hline & Male & $32(527)$ & $0.82(0.63-1.05)$ & \\
\hline \multirow{2}{*}{ Sample source } & Non-sentinel & $45(517)$ & 1 & 1 \\
\hline & Sentinel & $21(652)$ & $0.32(0.25-0.42)$ & $0.81(0.55-1.20)$ \\
\hline \multirow{2}{*}{ Seasonal influenza vaccination } & No & $35(781)$ & 1 & \multirow{2}{*}{ n.i. } \\
\hline & Yes & $25(24)$ & $0.61(0.24-1.55)$ & \\
\hline \multirow{2}{*}{ Any chronic underlying disease } & No & $48(435)$ & 1 & \multirow{2}{*}{ n.i. } \\
\hline & Yes & $69(55)$ & $2.42(1.32-4.41)$ & \\
\hline \multirow{2}{*}{ Diabetes } & No & $56(362)$ & 1 & \multirow{2}{*}{ n.i. } \\
\hline & Yes & $90(11)$ & $7.83(0.99-61.82)$ & \\
\hline \multirow{2}{*}{ Immunosuppression } & No & $49(465)$ & 1 & \multirow{2}{*}{ n.i. } \\
\hline & Yes & $78(18)$ & $3.61(1.17-11.12)$ & \\
\hline \multirow{2}{*}{ Cardiovascular disease } & No & $57(366)$ & 1 & \multirow{2}{*}{ n.i. } \\
\hline & Yes & $57(7)$ & $1.02(0.23-4.64)$ & \\
\hline \multirow{2}{*}{ Respiratory disease } & No & $72(228)$ & 1 & \multirow{2}{*}{ n.i. } \\
\hline & Yes & $80(15)$ & $1.53(0.42-5.59)$ & \\
\hline
\end{tabular}

CI: confidence interval; EEA: European economic area; EFTA: European Free Trade Association; EU: European Union; n.i.: not included in the final model. a Numbers in parentheses represent denominators for each category.

b Totals per each variable may be smaller than the total number of cases due to missing values.

c The final model included age, source of the sample and reporting country.

d p-value from likelihood ratio test comparing the model with and without age was $<0.08$.

\section{T A B L E 3}

Effect of oseltamivir resistance on clinical outcomes, data from five EU and EEA/EFTA countries, 2007-8 influenza season, sentinel networks $(n=790)$

\begin{tabular}{|c|c|c|c|c|c|}
\hline \multicolumn{2}{|l|}{ Outcome } & $\begin{array}{l}\% \text { oseltamivir-resistant } \\
\text { virus }^{\mathrm{a}} \\
\mathrm{N}: 138\end{array}$ & $\begin{array}{c}\% \text { oseltamivir-susceptible virus }{ }^{\mathrm{a}} \\
\mathrm{N}: 652\end{array}$ & $\begin{array}{l}\text { Crude risk ratios } \\
\qquad(95 \% \mathrm{CI})\end{array}$ & $\begin{array}{l}\text { Adjusted risk ratios } \\
(95 \% \mathrm{CI})^{\mathrm{b}}\end{array}$ \\
\hline \multirow{7}{*}{$\begin{array}{l}\text { Symptoms at } \\
\text { presentation }{ }^{c}\end{array}$} & Sudden onset & 97 (99) & 96 (459) & $1.01(0.81-1.26)$ & n.i. \\
\hline & Fever & 97 (99) & $96(381)$ & $1.01(0.81-1.26)$ & n.i. \\
\hline & Headache & $82(82)$ & 65 (165) & $1.25(0.92-1.69)$ & n.i. \\
\hline & Myalgia & 85 (130) & $83(456)$ & $1.01(0.82-1.25)$ & n.i. \\
\hline & Dry cough & $92(130)$ & $90(471)$ & $1.03(0.84-1.26)$ & n.i. \\
\hline & Sore throat & $66(79)$ & $53(163)$ & $1.23(0.87-1.74)$ & n.i. \\
\hline & Runny nose & $56(78)$ & $59(164)$ & $0.95(0.67-1.36)$ & n.i. \\
\hline \multirow{5}{*}{ Complications ${ }^{c}$} & Hospitalisation $^{d}$ & $2(123)$ & $1(247)$ & $1.34(0.22-8.01)$ & $1.25(0.21-7.58)$ \\
\hline & $\begin{array}{l}\text { Any clinical } \\
\text { complication }\end{array}$ & $8(120)$ & $5(244)$ & $1.69(0.73-3.92)$ & $1.59(0.68-3.71)$ \\
\hline & Pneumonia & $2(85)$ & $1(148)$ & $3.48(0.31-38.40)$ & $3.98(0.35-45.42)$ \\
\hline & Otitis & $3(86)$ & $4(149)$ & $0.87(0.22-3.46)$ & $0.94(0.23-3.84)$ \\
\hline & Death & $0(123)$ & $0(248)$ & & n.i. \\
\hline
\end{tabular}

CI: confidence interval; EEA: European economic area; EFTA: European Free Trade Association; EU: European Union; n.i.: not included in the final model. a Numbers in parentheses represent denominators for each category.

b Adjusted for age but not for the presence of chronic medical condition because of the high proportion of missing values for this variable.

c Each case may have presented multiple symptoms and developed multiple complications.

d Hospitalisation is included here for practical reasons but may have occurred for reasons other than clinical complications. 
a questionnaire by mail or telephone. In the UK, information was collected only on oseltamivir-resistant cases and there were no controls. GPs and hospital clinicians who had reported a case were contacted by national or local public health staff by telephone, and details were collected using a structured interview. In cases where clinicians were unable to provide the information, the patients were contacted directly.

\section{Data management and analysis}

Country-specific databases were shared with the ECDC for the final analysis. The databases were first analysed separately to detect differences in the results that would have to be considered in the pooled analysis. This was not possible for the UK data, which only included information on oseltamivir-resistant cases; however, these contributed to the pooled dataset. For each country, the prevalence of the various exposures in cases and controls was compared using contingency tables and the chi-squared test to check for statistical significance. Crude odds ratios were also computed. For the cohort approach, the prevalence (risk) of any of the considered clinical outcomes was calculated in exposed and unexposed individuals and the chi-squared test was used to check for statistical significance. Crude risk ratios were also computed. In order to allow for a pooled analysis of the five databases, they were merged into a unique database converting data from Access and Excel into STATA 10 format. Only variables collected by at least four of the five countries were retained in the final database.

The univariable analysis of the pooled database was conducted by using the procedures described above for the country-specific databases. The analysis of risk factors for severe influenza disease (cohort approach) was restricted to the population reported by sentinel surveillance systems. This was because individuals identified through non-sentinel sources are generally more likely to represent cases with more severe influenza and are thus already selected for the outcome of interest. By contrast, the analysis of risk factors for oseltamivir resistance was conducted first separately by source of the sample and then by combining the two populations. Multivariable analyses were conducted by using logistic regression to obtain adjusted odds ratios for the risk of being a case, and Poisson regression to obtain adjusted risk ratios for developing the outcomes of interest in the cohort analysis. Variables significant in univariable analyses $(p<0.05)$ were included in the initial multivariable models. The presence of effect modification between study country and each variable was checked, and in the absence of a significant interaction, country was treated as a potential confounder. A backward elimination procedure was used to build the final models. Despite the common protocol, covariates were not uniformly collected in the different studies. In order to determine the possible confounding effects of these variables, a sensitivity analysis was therefore conducted excluding studies one by one from the univariable analysis and the final multivariable models and comparing the results with those of all studies included.

Evaluation of resistance to neuraminidase inhibitors was carried out either at country level (when laboratory capacity was available) or by the Health Protection Agency (HPA) in London in collaboration with the WHO Collaborating Centre for Reference and Research on Influenza (WHO-CC). Assessment of resistance was through phenotypic analysis (IC50) or genotypic analysis (sequencing) for detection of the mutation H275Y. A subset of viruses tested for antiviral susceptibility both at HPA and NICs yielded $100 \%$ concordant results with respect to resistance status. IC50 and genetic testing performed on a subset of viruses were also $100 \%$ concordant [6].

\section{Results}

\section{Surveillance data}

The 2007-8 influenza season in Europe was initially dominated by type $A$ influenza viruses, and $96 \%$ of subtyped type A influenza viruses were $A(H 1)$ [6]. Type $B$ influenza viruses became dominant in week $8 / 2008$. For 30 countries in EISS, data on susceptibility of influenza $A(\mathrm{H} 1 \mathrm{~N} 1)$ viruses to oseltamivir were reported (Table 1$)$. From week 40/2007 to 27 August 2008, a total of 2,992 influenza $\mathrm{A}(\mathrm{H} 1 \mathrm{~N} 1)$ viruses were tested for oseltamivir resistance. Of these, $727(24.3 \%)$ were resistant to oseltamivir (Table 1 ). Resistance was reported in 22 countries and ranged from $1 \%(n=106)$ in Italy to $67 \%(n=274)$ in Norway (Table 1$)$. No resistance was found in eight countries, most of which were located in the central and eastern part of Europe (Bulgaria, Croatia, Czech Republic, Hungary, Latvia, Serbia, Slovakia and Turkey). However the period of testing and numbers of viruses tested were not representative and might have resulted in an underestimation of the real proportion of resistant viruses [6]. Oseltamivir-resistant viruses were detected in sentinel and non-sentinel patients, and the distribution varied by country (e.g. 20-30\% were reported from sentinel sources in the UK, the Netherlands and Norway, and around $80 \%$ in Germany and Luxembourg). Sixteen countries also reported case-based clinical information through the enhanced surveillance (Table 1) system as described in the methods section. However, the level of completeness of data was low in countries not conducting ad hoc epidemiological studies and therefore the analytical part of this article is based on the data provided by the five countries conducting such studies.

\section{Epidemiological studies}

Analysis by country

None of the main variables collected (age, sex, travel history, influenza vaccination, chronic medical condition) was significantly associated with an increased risk of infection with an oseltamivirresistant virus. Some of the variables analysed showed some effects that, although not statistically significant, deserve to be mentioned: In the Netherlands, individuals suffering from any kind of immunosuppression were more likely to be infected with an oseltamivir-resistant virus (odds ratio (OR): 5.5, 95\% confidence interval $(\mathrm{Cl}): 0.95$ to $32 ; \mathrm{p}=0.056)$. In addition, individuals reported through the sentinel system were less likely to be infected with a resistant virus (OR: $0.51,95 \% \mathrm{Cl}: 0.25$ to $1.04 ; \mathrm{p}=0.065$ ). In Norway, individuals aged between 18 and 64 years were more likely to be infected with a resistant virus than those younger than 18 years (OR: $1.84,95 \% \mathrm{Cl}: 1.09$ to $3.11 ; \mathrm{p}=0.022$ ).

Infection with a resistant virus was not significantly associated with an increased risk of pneumonia, hospitalisation or clinical complication in any of the five countries. In Luxembourg, the mean duration of influenza illness was longer in cases infected with oseltamivir-resistant virus than in oseltamivir-susceptible infections ( 10 and seven days, respectively; $p$-value $=0.025$ by $T$ test for the hypothesis of no difference between the two groups). There was no difference between the two groups with regards to the maximum temperature of fever $\left(39.3\right.$ versus $\left.39.3^{\circ} \mathrm{C}\right)$. In Norway, resistant cases were at higher risk of developing pneumonia (RR 3.15, 95\% $\mathrm{Cl}$ : 0.72 to 13.89 ); however, this association was not statistically significant. The results of the Norwegian study have recently been published as a separate article [11]. In the UK, the epidemiological information was only collected from the 36 cases with oseltamivirresistant infection, and bronchitis and pneumonia were the most commonly reported complications affecting six (17\%) and eight (22\%) cases, respectively. 
Results of the pooled data analysis

Following merging of the five national databases, information was available on 1,169 individuals with an influenza $A(H 1 N 1)$ infection, of which 373 (32\%) were oseltamivir-resistant. Information was incomplete for key variables such as presence of a chronic medical condition (58\% missing values) and hospitalisations (45\% missing values). The distribution of missing values was not substantially different between data coming from sentinel networks and data from non-sentinel sources. The proportion of missing information can be calculated by summing up the denominators of each variable reported in Tables 2 and 3 and comparing this with the total number of subjects reported in the Tables.

The analysis of risk factors for oseltamivir resistance was first undertaken separately by reporting source (sentinel and nonsentinel) and subsequently, since there were no relevant differences between the two sources, data from sentinel and non-sentinel sources were analysed together. By univariable analysis (Table 2), individuals aged between 18 and 64 years were almost twice as likely to have an infection with a resistant virus than those younger than 18 years (OR:1.93, 95\% Cl: 1.49 to 2.51 ). Only 10 individuals over the age of 64 years were reported and an association of resistance with older age could therefore not be ascertained. Those suffering from a chronic medical condition were 2.4 times more likely to be infected with a resistant virus than healthy individuals (OR:2.42, 95\% Cl: 1.32 to 4.41 ). Individuals identified through the sentinel network were less likely to be infected with a resistant virus than those identified through nonsentinel sources (OR:0.32, $95 \% \mathrm{Cl}: 0.25$ to 0.42 ).

Following multivariable analysis, none of these factors remained statistically significant. After adjusting for reporting country and source of the sample, the age-group of 18-64 year-olds was associated with a higher risk of being infected with an oseltamivirresistant virus than the younger age group (OR:1.39, $95 \% \mathrm{Cl}$ : 1.01 to 1.91 ), however the $p$ value from the likelihood ratio test comparing the models with and without the variable age was $<0.08$ (Table 2).

The cohort analysis to investigate the effect of oseltamivir resistance on disease severity and complications was restricted to subjects reported by the sentinel networks. There were no significant differences in symptoms at the time of sampling between exposed (oseltamivir-resistant) and non-exposed (oseltamivir-susceptible) patients (Table 3). The risk of influenza disease complications (hospitalisation, pneumonia, otitis media or death) was low for all subjects and did not significantly differ between exposed and non-exposed cases (Table 3).

The sensitivity analysis conducted on both univariable and multivariable models did not reveal substantial differences between countries. Where differences were detected, these only concerned the magnitude but not the direction of the effect. Tables with data of the full sensitivity analyses can be provided by the corresponding author upon request.

Four influenza-related deaths were reported among oseltamivirresistant cases detected through non-sentinel sources, of which three occurred in the UK and one in the Netherlands and none among oseltamivir-susceptible cases. These were two children (one newborn and one two year-old), one young adult and one person older than 65 years. With the exception of the newborn, all had a chronic medical condition that put them at higher risk of severe influenza and none had received influenza vaccination. None of these cases received oseltamivir treatment.

\section{Discussion}

This article provides a comprehensive analysis of the epidemiological information that was collected in Europe during the influenza season 2007-8 on individuals infected with an oseltamivir-susceptible or -resistant influenza $A(H 1 N 1)$ virus. Through the analysis of surveillance data and by combining the results of five national observational studies, we have provided evidence that infection with an oseltamivir-resistant $A(H 1 N 1)$ influenza virus was not related to any of the risk factors analysed. In particular, we did not identify any association between having a chronic medical condition and infection with an oseltamivirresistant virus. This finding is in contrast with previous observations where higher levels of oseltamivir resistance were mainly reported in vulnerable groups such as children and immunosuppressed individuals and in association with oseltamivir treatment [12$14]$, and is consistent with the results of a similar investigation conducted in the United States (US) [15] and Norway [11] during the same influenza season. A possible explanation for this finding could be that the oseltamivir-resistant influenza $A(H 1 N 1)$ viruses analysed in this study had become resistant by a process other than the selective pressure of oseltamivir treatment.

We observed a slightly higher risk of being infected with an oseltamivir-resistant virus among adults (18-64 years-old) compared with those younger than 18 years. We think that the most likely explanation for this finding is the confounding effect of different attitudes in different countries on when to consult a GP, and the fact that countries had a very different prevalence of oseltamivirresistant viruses. This hypothesis was supported by the reduction of the odds ratio towards unity that we observed when adjusting the effect of age for country reporting. Residual confounding that we were not able to adjust for may explain the borderline effect of age observed in the multivariable analysis.

Prior to the 2007-8 influenza season, studies conducted in animal models found that amino acid mutations in the neuraminidase protein causing oseltamivir drug resistance reduced the pathogenicity of the virus because of their effects on the neuraminidase enzyme function [16-20]. Our study found that individuals infected with an oseltamivir-resistant $A(H 1 N 1)$ virus experienced similar symptoms and risk of clinical complications as individuals infected with the same virus subtype susceptible to oseltamivir. Hence there was no clinical evidence that the resistant viruses differed from the susceptible viruses in terms of pathogenicity in humans. The four deaths reported in the UK and the Netherlands seem consistent with the incidence of influenzaassociated mortality in risk groups and it is unlikely that oseltamivir resistance played a role. However, it should be noted that the relatively small sample size might have prevented detection of significant differences in rare outcomes such as deaths.

All the viruses that were analysed genetically showed the same drug resistance mutation, the substitution of histidine by tyrosine at residue 275 ( $\mathrm{H} 275 \mathrm{Y})$ in the neuraminidase gene, which is known to confer high levels of resistance to oseltamivir in vitro [3], but has a reduced transmissibility [17]. However, the rare isolation of viruses carrying the $\mathrm{H} 275 \mathrm{Y}$ mutation from ill patients without known exposure to neuraminidase inhibitors [21] may indicate that some compensatory mutations within the neuraminidase, the haemagglutinin or other genes may be influencing virus 
transmissibility. Such compensatory mutations are likely to have determined the widespread circulation of fully transmissible and pathogenic oseltamivir-resistant influenza $A(H 1 N 1)$ viruses in Europe, although this still has to be ascertained. Limited variations in the susceptibility to neuraminidase inhibitors that occurred naturally over time (from 1997 to 2005) have been described for influenza $A(H 5 N 1)$ viruses, but do not seem to have clinical relevance so far [22].

The strength of our study is the consistency of results between countries and various sources of data (sentinel and non-sentinel), which validates the results of the pooled analysis. However, there are also important limitations that should be considered when interpreting the findings of this study. The main limitation is the high proportion of missing data for key variables. This was mainly due to the difficulties in collecting information on patients who had ILI months before the data collection started. In addition, data on follow-up outcomes may have been be inaccurate as they were collected from clinicians who were not necessarily aware of complications that may have occurred after they saw the patients. The study may also lack representativeness. In most of the countries, patients who underwent virological testing were selected neither randomly nor systematically, and clinicians may have preferentially tested patients with specific clinical characteristics or pre-existing conditions. In addition, since reporting for the sentinel cases was based on the standard case definition used for surveillance purposes, milder cases or those presenting with unusual clinical features may have been excluded from the study population. An information bias could have occurred if data for cases with oseltamivir-resistant virus infection were collected in more accurately than for cases with susceptible virus infection. We could not demonstrate this from the data available, but some of the participating countries that considered this issue found that clinicians were unaware of the oseltamivir resistance status of their patients at the time of the interview.

Even considering these limitations, this study has relevant public health implications. Subsequent results of global antiviral surveillance found that influenza $\mathrm{A}(\mathrm{H} 1 \mathrm{~N} 1)$ viruses resistant to oseltamivir have become predominant over susceptible strains, similarly to the evolution of circulating $A(H 3 N 2)$ viruses, most of which have become resistant to M2 inhibitors [23-26]. In Europe, preliminary results from the 2008-9 season show that while the $A(H 3 N 2)$ subtype predominated, almost all the influenza $A(H 1 N 1)$ viruses tested were oseltamivir-resistant [25]. Therefore, it is important that results from antiviral susceptibility surveillance are used to guide therapeutic decisions at an individual level. The US Centers for Disease Control and Prevention (CDC) issued recommendations for the use of antiviral medications in 2008-9. These took into account the strain-specific prevalence of oseltamivir resistance among circulating influenza $A$ viruses in the US, where resistant influenza $A(H 1 N 1)$ viruses predominated in the 2008-9 influenza season, and advised to use zanamivir or a combination of oseltamivir and rimantadine rather than oseltamivir alone when influenza $A(\mathrm{H} 1 \mathrm{~N} 1)$ virus infection or exposure is suspected [27]. These guidelines do not apply to Europe, where influenza A(H3N2) fully susceptible to neuraminidase inhibitors dominated during the season 2008-9 [28]. The findings of the present study suggest that influenza viruses naturally resistant to the currently available antivirals can rapidly emerge and circulate in the community. It is therefore important that new antiviral drugs against influenza are developed. Although the main tool for the prevention of influenza remains annual vaccination, there are circumstances when the use of antiviral drugs could play a pivotal role in preventing and reducing influenza morbidity. These would include the situation of a mismatch between the circulating and vaccine influenza strains, the control of outbreaks in special settings (e.g. nursing homes), or an influenza pandemic where vaccine is unlikely to be available until some months after the start of the pandemic.

The emergence of the $2009 \mathrm{H} 1 \mathrm{~N} 1$ influenza pandemic raised concerns over the possible emergence of oseltamivir resistance. Despite the wide use of neuraminidase inhibitors both for prophylaxis and treatment during the pandemic, oseltamivir resistance has so far only been detected sporadically and resistant viruses did not efficiently transmit in the community $[29,30]$. Diversification of national antiviral stockpiles to include different types of antivirals has been advised in some European countries $[1,31]$. The pandemic influenza $A(H 1 N 1) v$ virus is currently fully resistant to adamantanes but susceptible to both available neuraminidase inhibitors, zanamivir and oseltamivir [32].

In general, the unexpected emergence of high levels of oseltamivir resistance in Europe during the season 2007-8 highlights the evolving nature of the influenza virus and the requirement for a flexible approach to disease control including regular review and updating of treatment guidelines and pandemic plans [33].

What are the implications from this experience for the rapid, early assessment that is essential following the appearance of a pandemic [34]? Important lessons learnt are: 1) Reliance on referred specimens, especially from hospitalised or otherwise severe cases is likely to give a biased view of the pattern of infection in the community. 2) Multi-national approaches are more difficult once countries have started independent analytic approaches. It would be preferable for countries to develop and agree in advance on proposals (i.e. mock-up study protocols) to obtain the epidemiological information that is needed at the beginning of a pandemic to guide control measures. This is the approach being taken by the ECDC in collaboration with WHO and such plans should take into account the limitations identified in this study.

\section{Acknowledgements}

We are grateful to the Health Protection Agency Centre for Infection and the WHO Collaborating Centre in London for carrying out and rapidly sharing antiviral susceptibility tests in Europe. We are indebted with virologists, clinicians and epidemiologists of the participating countries for collecting and providing the information that made possible the current study. In particular we are grateful to the following persons: C Brown, WHO Regional Office for Europe; $P$ Huberty-Krau, Health Directorate and M Opp, National Health Laboratory, Luxembourg; L Jessop, R Pebody and PS Pilli, Health Protection Agency, UK; AB Osterhaus, G Rimmelzwaan, R van Beek, Erasmus Medical Centre, Rotterdam; G Donker, NIVEL Netherlands Institute for Health Services Research, Utrecht; M Koopmans, M Jonges, National Institute for Public Health and the Environment, Netherlands; S Brockmann, Robert KochInstitut, Germany.

At the time when the study was conducted, the VIRGIL project was receiving funding from the European Union FP6 Research Programme http://ec.europa.eu/research/health/influenza/proj13_en.aspx and EISS from ECDC. 


\section{References}

1. Meijer A, Lackenby A, Hay A, Zambon M. Influenza antiviral susceptibility monitoring activities in relation to national antiviral stockpiles in Europe during the winter 2006/2007 season. Euro Surveill. 2007;12(4):pii=698. Available from: http://www.eurosurveillance.org/ViewArticle.aspx?ArticleId=698

2. Lackenby A, Hungnes O, Dudman SG, Meijer A, Paget WJ, Hay AJ, et al. Emergence of resistance to oseltamivir among influenza $A(H 1 N 1)$ viruses in Europe. Euro Surveill. 2008;13(5):pii=8026. Available from: http://www.eurosurveillance.org/ ViewArticle.aspx?ArticleId $=8026$

3. McKimm-Breschkin J, Trivedi T, Hampson A, Hay A, Klimov A, Tashiro M, et al. Neuraminidase sequence analysis and susceptibilities of influenza virus clinical isolates to zanamivir and oseltamivir. Antimicrob Agents Chemother. 2003;47(7):2264-72.

4. European Centre for Disease Prevention and Control (ECDC). Antivirals and Antiviral Resistance - Influenza. Stockholm: ECDC. [Accessed 19 November 2009]. Available from: http://ecdc.europa.eu/en/healthtopics/Pages/Antivirals and_Antiviral_Resistance_Influenza.aspx

5. Influenza Project Team. Oseltamivir resistance in human seasonal influenza viruses (A/H1N1) in EU and EFTA countries: an update. Euro Surveill. 2008;13(6):pij=8032. Available from: http://www.eurosurveillance.org/ ViewArticle.aspx?ArticleId $=8032$

6. Meijer A, Lackenby A, Hungnes O, Lina B, van-der-Werf S, Schweiger B, et al. Oseltamivir-resistant influenza A (H1N1) virus, Europe, 2007-08 season. Emerg Infect Dis. 2009; 15(4):552-60.

7. Kramarz P, Monnet D, Nicoll A, Yilmaz C, Ciancio B. Use of oseltamivir in 12 European countries between 2002 and 2007--lack of association with the appearance of oseltamivir-resistant influenza $A(\mathrm{H} 1 \mathrm{~N} 1)$ viruses. Euro Surveill. 2009;14(5):pii=19112. Available from: http://www.eurosurveillance. org/ViewArticle.aspx?ArticleId $=19112$

8. Aguilera JF, Paget JW, Manuguerra JC, on behalf of the European Influenz Surveillance Scheme and EuroGROG. Survey of Influenza Surveillance Systems in Europe. Utrecht, the Netherlands: NIVEL. 2001.

9. Meijer A, Valette M, Manuguerra JC, Perez-Brena P, Paget J, Brown C, et al. Implementation of the community network of reference laboratories for human influenza in Europe. J Clin Virol. 2005;34(2):87-96.

10. Meijer A, Brown C, Hungnes O, Schweiger B, Valette M, van der Werf S, et al Programme of the Community Network of Reference Laboratories for Human Influenza to improve Influenza Surveillance in Europe. Vaccine. 2006;24(4446):6717-23.

11. Hauge SH, Dudman S, Borgen K, Lackenby A, Hungnes O. Oseltamivir-resistant influenza viruses A (H1N1), Norway, 2007-08. Emerg Infect Dis. 2009;15(2):15562.

12. Kiso M, Mitamura K, Sakai-Tagawa Y, Shiraishi K, Kawakami C, Kimura K, et al. Resistant influenza A viruses in children treated with oseltamivir: descriptive study. Lancet. 2004;364(9436):759-65.

13. Whitley RJ, Hayden FG, Reisinger KS, Young N, Dutkowski R, Ipe D, et al. Oral oseltamivir treatment of influenza in children. The Pediatric infectious disease journal. 2001;20(2):127-33.

14. Stephenson I, Democratis J, Lackenby A, McNally T, Smith J, Pareek M, et al. Neuraminidase Inhibitor Resistance after Oseltamivir Treatment of Acute Influenza A and B in Children. Clin Infect Dis. 2009;48(4):389-96.

15. Dharan NJ, Gubareva LV, Meyer JJ, Okomo-Adhiambo M, McClinton RC, Marshall $\mathrm{SA}$, et al. Infections with oseltamivir-resistant influenza $\mathrm{A}(\mathrm{H} 1 \mathrm{~N} 1)$ virus in the United States. Jama. 2009;301(10):1034-41.

16. McKimm-Breschkin JL. Resistance of influenza viruses to neuraminidase inhibitors--a review. Antiviral Res. 2000;47(1):1-17.

17. Herlocher ML, Truscon R, Elias S, Yen HL, Roberts NA, Ohmit SE, et al. Influenza viruses resistant to the antiviral drug oseltamivir: transmission studies in ferrets. J Infect Dis. 2004;190(9):1627-30.

18. Hurt AC, Ho HT, Barr I. Resistance to anti-influenza drugs: adamantanes and neuraminidase inhibitors. Expert Rev Anti Infect Ther. 2006;4(5):795-805.

19. Yen HL, Herlocher LM, Hoffmann E, Matrosovich MN, Monto AS, Webster RG, et al. Neuraminidase inhibitor-resistant influenza viruses may differ substantially in fitness and transmissibility. Antimicrob Agents Chemother. 2005;49(10):407584.

20. Zurcher T, Yates PJ, Daly J, Sahasrabudhe A, Walters M, Dash L, et al. Mutations conferring zanamivir resistance in human influenza virus N2 neuraminidases compromise virus fitness and are not stably maintained in vitro. J Antimicrob Chemother. 2006;58(4):723-32.

21. Monto AS, McKimm-Breschkin JL, Macken C, Hampson AW, Hay A, Klimov A, et al. Detection of influenza viruses resistant to neuraminidase inhibitors in global surveillance during the first 3 years of their use. Antimicrob Agents Chemother. 2006;50(7):2395-402.

22. Rameix-Welti MA, Agou F, Buchy P, Mardy S, Aubin JT, Veron M, et al. Natural variation can significantly alter the sensitivity of influenza $A(\mathrm{H} 5 \mathrm{~N} 1)$ viruses to oseltamivir. Antimicrob Agents Chemother. 2006;50(11):3809-15.
23. Deyde VM, Xu X, Bright RA, Shaw M, Smith CB, Zhang Y, et al. Surveillance of resistance to adamantanes among influenza $A(H 3 N 2)$ and $A(H 1 N 1)$ viruses isolated worldwide. J Infect Dis. 2007;196(2):249-57.

24. European Centre for Disease Prevention and Control (ECDC). Monitoring of Influenza antiviral resistance in EU during 2008-09 season. Stockholm: ECDC. [Accessed 19 November 2009]. Available from: http://ecdc.europa.eu/en/ healthtopics/Pages/Antivirals_and_Antiviral_Resistance_Influenza_Weekly_ Updates.aspx

25. Goddard N, Zucs P, Ciancio B, Plata F, Hungnes O, Mazick A, et al. Start of the influenza season 2008-9 in Europe - increasing influenza activity moving from West to East dominated by A(H3N2). Euro Surveill. 2009;14(3):pii=19097. Available from: http://www.eurosurveillance.org/ViewArticle.aspx?ArticleId=19097

26. World Health Organization (WHO). Influenza A(H1N1) virus resistance to oseltamivir - 2008/2009 influenza season, northern hemisphere. Geneva: WHO. [Accessed 19 November 2009]. Available from: http://www.who.int/csr/disease/ influenza/H1N1webupdate20090318\%20ed_ns.pdf

27. Centers for Disease Control and Prevention (CDC). CDC Issues Interim Recommendations for the Use of Influenza Antivirals in the Setting of Oseltamivir Resistance among Circulating Influenza A (H1N1) Viruses, 200809 Influenza Season. Atlanta: CDC. [Accessed 19 November 2009]. Available from: http://www2a.cdc.gov/HAN/ArchiveSys/ViewMsgV.asp?AlertNum=00279

28. ECDC Influenza news. Public Health Developments: Seasonal Influenza Activity and Antiviral Resistance- United States (28 September - 29 November 2008). 2008. Stockholm: ECDC; 18 December 2008. Available from: http://www.ecdc. europa.eu/en/healthtopics/Lists/Influenza\%20Newsletter/DispForm.aspx?ID=10 $2 \&$ Source $=$ http\%3A\%2F\%2Fwww.ecdc.europa.eu\%2Fen\%2Fhealthtopics\%2FLists\%2 FInfluenza\%2520Newsletter\%2FAllItems.aspx\%3FPaged\%3DTRUE\%26p_ID\%3D100\% 26View\%3D\%257b19207E2B\%252dCCA9\%252d4968\%252d9706\%252dE26582ADE374\%25 $7 \mathrm{~d} \% 26$ FolderCTID\%3D0x012001\%26PageFirstRow\%3D101

29. Centers for Disease Control and Prevention (CDC). Oseltamivir-resistant 2009 pandemic influenza A (H1N1) virus infection in two summer camper receiving prophylaxis--North Carolina, 2009. MMWR Morb Mortal Wkly Rep. 2009;58(35):969-72.

30. Leung TW, Tai AL, Cheng PK, Kong MS, Lim W. Detection of an oseltamivirresistant pandemic influenza A/H1N1 virus in Hong Kong. J Clin Virol. 2009:46(3):298-9.

31. European Centre for Disease Prevention and Control (ECDC). Influenza News. Public health developments. A Member State Independent Expert Committee (UK - Scientific Pandemic Influenza) publish an expert opinion on the content and deployment of an extended antiviral stockpile. [Accessed 19 November 2009]. Available from: http://ecdc.europa.eu/en/healthtopics/Lists/Influenza\%20 Newsletter/DispForm.aspx?ID=6

32. European Centre for Disease Prevention and Control (ECDC). Surveillance Report. Weekly influenza surveillance overview, October 20, 2009. Stockholm: ECDC. Available from: http://ecdc.europa.eu/en/activities/surveillance/EISN/ Newsletter/091016 EISN Weekly_Influenza_Surveillance_Overview.pdf (accessed on 28/10/2009). 2009.

33. Fleming DM, Elliot AJ, Meijer A, Paget WJ. Influenza virus resistance to oseltamivir: what are the implications? Eur J Public Health. 2009;19(3):238-9.

34. Nicoll $A$, on behalf of the Influenza Project Team. Public Health Measures in an Influenza Pandemic - the importance of surveillance. Euro Surveill. 2007:12(44):pii=3300. Available from: http://www.eurosurveillance.org/ ViewArticle.aspx?ArticleId $=3300$ 